\title{
Genetics and the brain: many pathways to enlightenment
}

\author{
Michael C. O’Donovan • Michael J. Owen
}

Published online: 25 June 2009

(C) Springer-Verlag 2009

This special review issue on Neurological and Psychiatric Diseases and Traits focuses upon a number of phenotypes which, while very different, result from impairment of, or variation in, some aspect of brain function. The brain is widely regarded as one of the most challenging subjects for biomedical research. Its anatomical organization is highly complex, its functional organization is poorly understood, and notwithstanding advances in neuroimaging, it remains relatively inaccessible to direct study in vivo. Moreover, whereas the wider functions of most organs can be understood in basic biochemical and/or physical terms, this is not often the case for the brain. For example, the genesis of the higher order brain functions that are often central to brain disorders such as self-awareness, perception, thought, mood, and volition cannot be readily understood in terms of fundamental molecular mechanisms. In many cases, to these difficulties can be added a deficiency of relevant molecular and phenotypic model systems, the benefits of which are illustrated herein by the review of Down syndrome (Patterson 2009). While brain disorders are undeniably challenging targets for biomedical research, they are also amongst the most important, the brain being central to every aspect of human life.

With its role in human adaptability and survival, it would be remarkable if traits that result from variation in brain function were not influenced in part by genes. However, even in fairly recent times, the hypothesis that genes contribute to variation in normal cognitive and behavioural traits has been controversial. As illustrated by the articles

M. C. O’Donovan $(\bowtie) \cdot$ M. J. Owen

Department of Psychological Medicine and Neurology, School of Medicine, MRC Centre for Neuropsychiatric Genetics and Genomics, Heath Park, Cardiff CF23 6BQ, UK e-mail: odonovanmc@cf.ac.uk on human intelligence (Deary et al. 2009) and on aggression (Craig and Halton 2009), there is now convincing evidence (some of it old) that genes are involved, and the same is true for many other cognitive and behavioural traits. Genetic methods, therefore, offer one possible route to trying to understand normal variation in brain function. The other reviews have as their subjects heritable brain-related phenotypes that are generally thought of as 'disorders'; like intelligence and aggression, some of these can alternatively be conceptualized as quantitative traits, as illustrated in the review of ADHD by Franke and colleagues.

In addition to the general difficulties alluded to above, many of the disorders reviewed herein also have features that make genetic studies problematic. Discussed in detail in the review of psychosis (O'Donovan et al. 2009), often the disorders cannot be classified based upon any biologically validated methodology. Consequently, the power of genetic studies is likely to be compromised by sub-optimal phenotypic definitions and extensive heterogeneity. In some cases, it has been possible to overcome this by identifying relatively homogenous groups based upon the presence of other syndromic features and/or by studying large single families co-segregating major genes, as exemplified in the reviews of epilepsy (Andrade 2009) and migraine (de Vries et al. 2009). However, such approaches are not currently applicable to the majority of people with those disorders, or to individuals with other common neurological and psychiatric phenotypes. Another approach that has met with considerable success in identifying genes that contribute to Mental Retardation has been the study of balanced translocations (Vandeweyer and Kooy 2009) and other chromosomal abnormalities. Such gross abnormalities may not contribute substantially to typical forms of common disorders. However, studies of smaller chromosomal abnormalities in the form of rare copy number variants are beginning 
to shed light on disease mechanisms in apparently typical cases of schizophrenia as well as autism, epilepsy, and mental retardation (O'Donovan et al. 2009).

While the relative contributions of rare and common risk variants are unknown, it seems likely that most common disorders will involve both, and that for each disorder, numerous loci will be involved. In the absence of information about the position of susceptibility genes through the study of chromosomal abnormalities or linkage, researchers for many brain phenotypes have had to rely on a candidate gene approach. For common alleles, the emergence of genome wide association (GWA) technology has removed this reliance, and it is expected that within the next few years, the emergence of cheap whole genome sequencing will do the same for rare allele detection. With respect to common alleles, it is now evident from the GWA studies that have been performed in other phenotypes that the typical effect sizes conferred by those alleles is very small, and that power to detect real effects with the samples deployed in candidate gene studies to date has been much lower than many would have expected. It is, therefore, unclear whether the failure of the candidate gene strategy alluded to by many of the reviews (O'Callaghan et al. 2009; Gizer et al. 2009; Craig and Halton 2009) reflects lack of power or poor choice of candidates. In the review on alcohol dependence, Gelernter and Kranzler (2009) provide examples where candidate gene approaches have been successful. However, it is probably the case that those candidates (e.g. alcohol metabolizing enzymes) had, a priori, a much more direct functional link to the phenotype than of the most candidate genes that have been studied in other disorders, as attested by the same handful of genes repeatedly turning up as candidates for many of the phenotypes that are the subject of the present set of reviews.

If the relative failure of candidate gene studies primarily reflects underpowered samples or poorly chosen candidates rather than genetic architectures that do not include common alleles, then SNP-based genome wide association studies (GWAS) based upon larger samples should provide a fruitful alternative. Few GWAS studies have been performed for the phenotypes that are the subject of the present reviews. Where they have been, as discussed in the reviews of psychosis, ADHD and alcohol dependence (O'Donovan et al. 2009; Franke et al. 2009; Gelernter and Kranzler 2009), they have provided novel insights into disease mechanisms. This suggests that the difficulties notwithstanding, many other brain-related phenotypes will similarly benefit from the GWAS approach, with the proviso that adequate sample sizes are studied.

Diagnostic challenges, small effect sizes, and little or no knowledge of pathophysiology have all been major impediments to progress. This is not unique to disorders affecting the brain, but their combined effects here are perhaps at their most acute. Nevertheless, the reviews provide clear evidence that these difficulties are not insurmountable. They illustrate that no single approach is appropriate to all cases and that it is important to deploy all available molecular genetic tools, old and new. Given the uncertainty about the genetic architectures of the disorders, it is unclear from what direction most breakthroughs will come, but no doubt further successes will emerge as it becomes possible to study the genome in ever increasing detail. Meanwhile, for many of the phenotypes discussed, it is clearly a priority that those newer tools which exist are now applied to samples that are large enough to detect weak genetic effects, and which are sufficiently well phenotyped to allow post hoc dissection of underlying heterogeneity. At the same time, the reviews also show that gains that can be made by studying the rare and unusual. With the technologies that are now available and those that are on the horizon, we have little doubt that many more pathogenic mutations and susceptibility alleles for neurological and psychiatric disorders will be detected in the next few years, but such is their complexity that it will take many years to assemble anything other than a very incomplete picture of the genetic basis of these disorders. Nevertheless, in most cases, even partial pictures constitute major advances on current knowledge of pathophysiology, and should provide novel insights that can be translated into improved patient care.

\section{References}

Andrade D (2009) Genetic basis in epilepsies caused by malformation of cortical development and in those with structurally normal brain. Human Genet. doi:10.1007/s00439-009-0702-1

Craig I, Halton K (2009) Genetics of human aggressive behaviour. Human Genet. doi:10.1007/s00439-009-0695-9

de Vries B, Frants RR, Ferrari MD, van den Maagdenberg AMJM (2009) Molecular genetics of migraine. Human Genet. doi:10.1007/s00439-009-0684-Z

Deary IJ, Johnson W, Houlihan LM (2009) Genetic foundations of human intelligence. Human Genet. doi:10.1007/s00439-009-0655-4

Franke B, Neale BM, Faraone SV (2009) Genome-wide association studies in ADHD. Human Genet. doi:10.1007/s00439-009-0663-4

Gelernter J, Kranzler H (2009) Genetics of alcohol dependence. Human Genet. doi:10.1007/s00439-009-0701-2

Gizer IR, Ficks C, Waldman ID (2009) Candidate gene studies of ADHD: a meta-analytic review. Human Genet. doi:10.1007/ s00439-009-0694-x

O'Callaghan ME, MacLennan AH, Haan EA, Dekker G, The South Australian Cerebral Palsy Research Group (2009) The genomic basis of cerebral palsy: a HuGE systematic literature review. Human Genet. doi:10.1007/s00439-009-0638-5

O’Donovan M, Craddock NJ, Owen MJ (2009) Genetics of psychosis; insights from views across the genome. Human Genet. doi:10.1007/s00439-009-0703-0

Patterson D (2009) Molecular genetic analysis of down syndrome. Human Genet. doi:10.1007/s00439-009-0696-8

Vandeweyer G, Kooy RF (2009) Balanced translocations in mental retardation. Human Genet. doi:10.1007/s00439-009-0661-6 